\section{Portuguese Journal of Public Health}

Abrantes A. 51

Abrantes, P. 18

Aguiar, P. 51

Almeida, C. 26

Campos Fernandes, A. 18

Correia, A. 26

da Costa, E.M. 18

da Costa, N.M. 18

de Carvalho, M.J.L. 32

Dias, S. 32, 42

Duarte, A. 11

Duarte, V. 32

Duarte-Ramos, F. 18

Filipe, C. 11

Fonseca, I. 11

França, D. 26
Gama, A. 32, 42

Gaspar, R. 18

Guerreiro, A.E. 32

Keygnaert, I. 32

Laires, P.A. 42

Leite, A. 18

Lobo, R. 26

Lopes, S. 11

Marquês, D. 11

Matias, A. 32

Mendonça-Galaio, L. 26

Mestre, R. 11

Middleton, J. 1

Moniz, M. 4, 42

Nunes, C. 4
Pedro, A.R. 32, 42

Quintas, J. 32

Raposo, J. 26

Ricoca Peixoto, V. 18, 51

Rocha, J. 11, 18

Sacadura-Leite, E. 26

Santana, R. 11

Serranheira, F. 26

Shapovalova, O. 26

Soares, J. 26

Soares, P. 4, 11, 42

Sousa, J. 11

Sousa, P. 18, 51

Sousa-Uva, A. 26

Teixeira, M. 11

Vieira, A. 51

\title{
Subject Index Vol. 38, Suppl. 1, 2020
}

Access to care 11

Community-based survey 42

COVID-19 4, 11, 18, 26, 42, 51

- pandemic 32

Domestic violence 32

Epidemiology 4, 51

Excess mortality 51

Health management software 26

- services 11
Healthcare workers 26

Help seeking 32

Hospitalizations 11

Municipal level 18

(Non-)Linear model 18

Occupational health 26

Online survey 32

Pandemics 18

Portugal 11, 51
Public health 32

- - surveillance 51

Quantitative methods 42

Relative risk 4

SARS-CoV-2 26

Social opinion 42

- perception 42

Spatial distribution 4

Transmission dynamics 4 karger@karger.com www.karger.com/pjp

Karger

̧OPEN ACCESS
(C) 2021 The Author(s.) Published by S. Karger AG, Basel on behalf of Escola Nacional de Saúde Pública

This is an Open Access article licensed under the Creative Commons Attribution-NonCommercial-4.0 International License (CC BY-NC) (http://www.karger.com/Services/OpenAccessLicense), applicable to the online version of the article only. Usage and distribution for commercial purposes requires written permission. 